\title{
Um inimigo silencioso: óbitos por suicídio no Estado da Paraíba no período de 2015-
}

\section{9}

\author{
A silent enemy: suicide deaths in the State of Paraíba in the period 2015-2019 \\ Un enemigo silencioso: muertes por suicidio en el Estado de Paraíba en el periodo de 2015-2019
}

Recebido: 22/07/2021 | Revisado: 30/07/2021 | Aceito: 03/08/2021 | Publicado: 08/08/2021

\author{
Dalila Camêlo Aguiar \\ ORCID: https://orcid.org/0000-0002-2901-4639 \\ Universidade de Granada, Espanha \\ E-mail: dalilacamelo@correo.ugr.es \\ Edwirde Luiz Silva Camêlo \\ ORCID: https://orcid.org/0000-0003-3686-927X \\ Universidade Estadual da Paraíba, Brasil \\ E-mail: edwirde@uepb.edu.br \\ Fernanda de Oliveira Lima \\ ORCID: https://orcid.org/0000-0002-8263-1759 \\ Universidade Estadual da Paraíba, Brasil \\ E-mail: fer.lima2812@gmail.com
}

\begin{abstract}
Resumo
Objetivo: analisar o perfil epidemiológico dos casos de suicídio na Paraíba/Brasil, em 2015 a 2019. Métodos: coletouse dados do Sistema de Informação sobre Mortalidade (SIM) e foi analisado segundo as categorias de lesões autoinfligidas intencionalmente (X60 a X84) da Classificação Internacional de Doenças (CID-10), examinando-se as distribuições por gênero, raça, faixa etária, escolaridade e local de residência (por microrregiões e mesorregiões). Resultados: foram registrados 1.140 mortes, sendo principalmente de pessoas com idade ativa de trabalho de 20 a 59 anos $(73,4 \%)$. Majoritariamente do sexo masculino $(78,0 \%)$, com ligeira predominância na faixa etária de 30 a 39 anos $(20,0)$, solteiros (mais de $25 \%$ ), de cor parda (mais de $60 \%$ ) com 4 a 7 anos de estudos (6,3\%); o principal meio utilizado foi a categoria X70 (enforcamento, estrangulamento e sufocação) com 83,0\%. Conclusão: a mortalidade por suicídio continua crescendo na Paraíba e varia em função do gênero e cor, sendo mais elevada nas microrregiões de João Pessoa e Campina Grande. Assim, são necessárias estratégias de promoção, prevenção e acompanhamento no âmbito da ação social e dos cuidados de saúde mental, com importantes variações regionais.
\end{abstract}

Palavras-chave: Óbitos por suicídio; Estado da Paraíba; Gênero; Raça; Séries temporais.

\begin{abstract}
Objective: to analyze the epidemiological profile of suicide cases in Paraíba/Brazil, from 2015 to 2019. Methods: data were collected from the Mortality Information System (MIS) and analyzed according to the categories of intentional self-harm (X60 to X84) of the International Classification of Diseases (ICD-10), examining the distributions by gender, race, age group, education and region of residence. Results: a total of 1,140 deaths were registered, mainly of people with active working age from 20 to 59 years (73.4\%). The majority was male (78.0\%), with a slight predominance in the 30-39 age group (20.0\%), single (more than 25\%), brown (more than 60\%), with 4 to 7 years of schooling (6.3\%); the primary method used was category X70 (hanging, strangulation and suffocation) with 83.0\%. Conclusion: suicide mortality continues to grow in Paraíba and varies according to gender and colour, being higher in the microregions of João Pessoa and Campina Grande. Thus, are necessary promotion, prevention and monitoring strategies in social action and mental health care, with significant regional variations.
\end{abstract}

Keywords: Suicide deaths; State of Paraíba; Gender; Race; Time series.

\section{Resumen}

Objetivo: Analizar el perfil epidemiológico de los casos de suicidio en Paraíb/Brasil, en 2015 a 2019. Métodos: los datos fueron recolectados del Sistema de Información de Mortalidad (SIM) y se analizó el suicidio según las categorías de lesiones autoinfligidas intencionadamente (X60 a X84) de la Clasificación Internacional de Enfermedades (CIE-10), examinando las distribuciones por género, raza, grupo de edad, educación y región de residencia (microrregiones y mesorregiones). Resultados: se registraron 1.140 defunciones, principalmente de personas en edad laboral activa de 20 a 59 años $(73,4 \%)$. En su mayoría hombres $(78,0 \%)$, con leve predominio en el grupo de edad de 30 a 39 años (20,0), solteros (más del 25\%), morenos (más del 60\%) con 4 a 7 años de estudios $(6,3$ $\%$ ); el principal medio utilizado fue la categoría X70 (ahorcamiento, estrangulamiento y asfixia) con 83,0\%. Conclusión: la mortalidad por suicidio sigue creciendo en Paraíba y varía según el género y color de piel, siendo 
mayor en las microrregiones de João Pessoa y Campina Grande. Por tanto, se requieren estrategias de promoción, prevención y seguimiento en el ámbito de la acción social y la atención de la salud mental, con importantes variaciones regionales.

Palabras clave: Muertes por suicidio; Estado de Paraíba; Género; Raza; Series temporales.

\section{Introdução}

O suicídio constitui-se em um dos mais antigos temas relacionados à saúde dos indivíduos e à forma como são afetados pelas sociedades e coletividades nas quais vivem. Em termos históricos, sua relevância no plano social pode ser identificada desde a Grécia antiga. Em tempos modernos, ao menos desde o século XVIII, tem sido tratado como fenômeno social e segundo perspectivas históricas, sociológicas, econômicas e filosóficas (Ribeiro \& Moreira, 2018).

A definição para o suicídio pode ser entendida como o resultado de comportamentos auto-infligidos que têm como intenção a própria morte (Kolves \& De Leo, 2014). Essa forma de violência autoinflingida resulta da interação de múltiplos fatores: biológicos, psicológicos, socioculturais e ambientais (CEVS, 2018). No entanto, na maioria das vezes, as tentativas e mortes por suicídio estão ligados a problemas de saúde mental (Cavanagh et al., 2003) que, se diagnosticados e tratados apropriadamente, podem levar a uma diminuição das ocorrências. A depressão, por exemplo, apresenta óbvia relação com as tentativas de suicídio em diferentes faixas etárias (Chachamovich et al., 2009; Cavalcante et al., 2013; Teismann et al., 2018; del Amo et al., 2019).

No mundo mais de 800.000 pessoas cometem suicídio todos os anos, representando uma morte a cada 40 segundos. É a segunda principal causa de morte na faixa etária de 15 a 29 anos (OMS, 2018), pelo que este fenômeno tornou-se um grave problema de saúde pública em muitos países, não ocorrendo apenas em países de alta renda, mas sendo um fenômeno em todas as regiões do mundo. Em 2019, 70\% desses casos aconteceram em países com vulnerabilidade social (WHO, 2019).

No Brasil, em 2016 o número de suicídios aumentou 2,3\% em 1 ano comparado ao ano anterior, e teve 1 caso a cada 46 minutos, sendo a quarta causa de morte entre jovens de 15 a 29 anos (Brasil, 2017). Embora o governo estima que o número seja ainda maior devido à subnotificação nos registros. No ranking de países com maior incidência de suicídios, o Brasil ocupava a oitava posição ultrapassando o número de 12 mil casos anuais de mortes autoprovocadas intencionalmente (OMS, 2018).

No Nordeste, as notificações de suicídio na região aparecem de forma preocupante, entre 2003 a 2013, os Estados da Paraíba, Piauí e Sergipe, mais que triplicaram seus quantitativos (Ceccon, 2014). De acordo com Silva et al (2021) a região Nordeste apresentou crescimento significativo da mortalidade por suicídio em 2008 a 2018.

De acordo com o Sistema de Informação sobre Mortalidade da Secretaria Estadual de Saúde (SIM/SES) a Paraíba registrou um caso de suicídio, a cada 34 horas. O dado foi divulgado pelo Ministério Público da Paraíba (Brasil, 2019). Conforme estudo realizado por Lavor et al. 2020, em que analisou dados de 2013 a 2017, observou que o número de mortes causadas por autoeliminação voluntária no Estado vem aumentando a cada ano.

Considerando o suicídio um importante problema de saúde pública ainda pouco estudado no Estado da Paraíba, o presente estudo tem como objetivo analisar o perfil epidemiológico dos casos de suicídio na Paraíba, Brasil, em 2015 a 2019.

\section{Materiais e Métodos}

Neste trabalho, realizou-se um estudo descritivo, de série temporal, investigando-se o perfil das pessoas que cometeram suicídio, por gênero, raça, faixa etária, escolaridade e local de residência (por microrregiões e mesorregiões) no período de 2015 a 2019 no Estado da Paraíba.

Os dados utilizados neste trabalho foram coletados das estatísticas de óbitos causado por lesões autoprovocadas intencionalmente de acordo com as causas X60 a X84 da 10ª Classificação Internacional de Doenças (CID-10) pelo Sistema de 
Informações de Mortalidade (SIM) em março de 2021. Posteriormente, foram analisados no programa estatístico R versão 3.5.2, sendo os pacotes utilizados ggplot2 (Wickham, 2016) para criação de gráficos baseado na gramática de gráfico e leaflet (Cheng et al, 2021) que usa uma biblioteca JavaScript para criação de mapas interativos.

\section{Resultados e Discussão}

Na Paraíba, o número de óbitos por suicídio no período de 2015 a 2019 foi de 1.140 mortes. A Tabela 1 apresenta o quantitativo e percentual por tipo de lesão autoprovocadas intencionalmente (o suicídio) de acordo com o gênero.

Tabela 1. Óbito por suicídio de acordo com as lesões autoprovocadas intencionalmente CID-10 (X60 a X84) e gênero. Paraíba, Brasil, 2015-2019.

\begin{tabular}{|c|c|c|c|c|}
\hline \multicolumn{2}{|c|}{ Categoria CID-10 } & \multirow{2}{*}{$\begin{array}{l}\text { Masc. } \\
(\%)\end{array}$} & \multirow{2}{*}{$\begin{array}{l}\text { Fem. } \\
(\%)\end{array}$} & \multirow{2}{*}{$\begin{array}{l}\text { Total } \\
(\%) \\
1(0,09) \\
\end{array}$} \\
\hline 0 & Analgésicos, antipiréticos e anti-reumáticos, não-opiáceos (X60) & & & \\
\hline \multirow{4}{*}{ 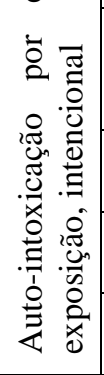 } & $\begin{array}{l}\text { Drogas anticonvulsivantes [antiepilépticos] sedativos, hipnóticos, } \\
\text { antiparkinsonianos e psicotrópicos não classificados em outra parte } \\
\text { (X61) }\end{array}$ & $3(60)$ & $2(40)$ & $5(0,44)$ \\
\hline & $\begin{array}{l}\text { narcóticos e psicodislépticos [alucinógenos] não classificados em outra } \\
\text { parte (X62) }\end{array}$ & $1(100)$ & $0(0)$ & $1(0,09)$ \\
\hline & $\begin{array}{l}\text { Outras substâncias farmacológicas de ação sobre o sistema nervoso } \\
\text { autônomo (X63) }\end{array}$ & $1(20)$ & $4(80)$ & $5(0,44)$ \\
\hline & $\begin{array}{l}\text { Outras drogas, medicamentos e substâncias biológicas e às não } \\
\text { especificadas (X64) }\end{array}$ & $13(39)$ & $20(61)$ & $33(2,89)$ \\
\hline \multirow{5}{*}{ 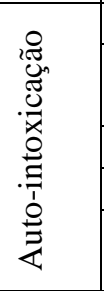 } & Voluntária por álcool (X65) & $9(100)$ & $0(0)$ & $9(0,79)$ \\
\hline & $\begin{array}{l}\text { Intencional por solventes orgânicos, hidrocarbonetos halogenados e } \\
\text { seus vapores (X66) }\end{array}$ & $2(100)$ & $0(0)$ & $2(0,18)$ \\
\hline & Intencional por outros gases e vapores (X67) & $0(0)$ & $1(100)$ & $1(0,09)$ \\
\hline & Por e exposição, intencional, a pesticidas (X68) & $65(59)$ & $46(41)$ & 9,74 \\
\hline & $\begin{array}{l}\text { Por e exposição, intencional, a outros produtos químicos e substâncias } \\
\text { nocivas não especificadas (X69) }\end{array}$ & $10(59)$ & $7(41)$ & $17(1,49)$ \\
\hline \multirow{13}{*}{ 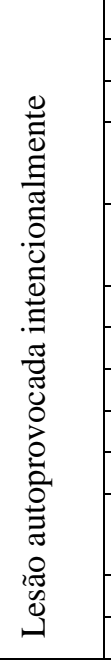 } & Enforcamento, estrangulamento e sufocação (X70) & $696(83)$ & $140(17)$ & $836(73,33)$ \\
\hline & Afogamento e submersão (X71) & $2(100)$ & $0(0)$ & 0,18 \\
\hline & Disparo de arma de fogo de mão (X72) & $18(82)$ & $4(18)$ & $22(1,93)$ \\
\hline & $\begin{array}{l}\text { Disparo de espingarda, carabina, ou arma de fogo de maior calibre } \\
\text { (X73) }\end{array}$ & $1(50)$ & $1(50)$ & $2(0,18)$ \\
\hline & $\begin{array}{l}\text { Disparo de outra arma de fogo e de arma de fogo não especificada } \\
\text { (X74) }\end{array}$ & $26(90)$ & $3(10)$ & $29(2,54)$ \\
\hline & Pela fumaça, pelo fogo e por chamas (X76) & $4(57)$ & $3(43)$ & $7(0,61)$ \\
\hline & Vapor de água, gases ou objetos (X77) & $1(100)$ & $0(0)$ & $1(0,09)$ \\
\hline & Objeto cortante ou penetrante (X78) & $5(83)$ & $1(17)$ & $6(0,53)$ \\
\hline & Objeto contundente (X79) & $0(0)$ & $1(100)$ & $1(0,09)$ \\
\hline & Precipitação de um lugar elevado (X80) & $29(69)$ & $13(31)$ & $42(3,68)$ \\
\hline & $\begin{array}{l}\text { Precipitação ou permanência diante de um objeto em movimento } \\
\text { (X81) }\end{array}$ & $1(33)$ & $2(67)$ & $3(0,26)$ \\
\hline & Impacto de um veículo a motor (X82) & $1(50)$ & $1(50)$ & $2(0,18)$ \\
\hline & Meios não especificados (X84) & $1(50)$ & $1(50)$ & $2(0,18)$ \\
\hline \multicolumn{2}{|l|}{ Total } & $889(78)$ & $251(22)$ & $1140(100)$ \\
\hline
\end{tabular}

Obs.: As categorias Dispositivos explosivos (X75) e outros meios especificados (X83) não registraram óbitos.

Fonte: Elaboração própria a partir de dados do SIM/DATASUS/MS.

Conforme a Tabela 1, 78\% dos óbitos por suicídio correspondem ao gênero masculino, com média de 177,8 óbitos por ano e $22,0 \%$ são femininos com média de 50,2. Estes resultados estão em conformidade com os apontados para o Brasil (Machado \& dos Santos, 2015), outros Estados e no Distrito Federal (Macente \& Zandonade, 2011; Bando, et al., 2012; 
Sehnem \& Palosqui, 2014; Carmo et al., 2018; Baére \& Zanello, 2018; Beringuel et al., 2020) e também consonante com a literatura internacional (Romero-Pimentel et al., 2018; Nunes, 2018; Kootbodien et al., 2020).

Majoritariamente o tipo de lesão mais frequente entre os óbitos por suicídio foi CID-10 (X70), ou seja, 73,33\% ocorrem por enforcamento, estrangulamento e sufocação, sendo 696 (83\%) homens e 140 (17\%) mulheres. Em segundo lugar, o tipo de lesão mais recorrente são as pesticidas (X68) com 9,74\% (59\% para homens e 41\% para mulheres). Entre os suicídios autocometidos por homens, também é recorrente o disparo de arma de fogo (X72 e X74), com 82\% e 90\%, respectivamente.

Em seguida, na Figura 1 temos a distribuição de óbitos por suicídio de acordo com ano e gênero.

Figura 1. Óbitos por suicídio por ano segundo o gênero. Paraíba, Brasil, 2015-2019.

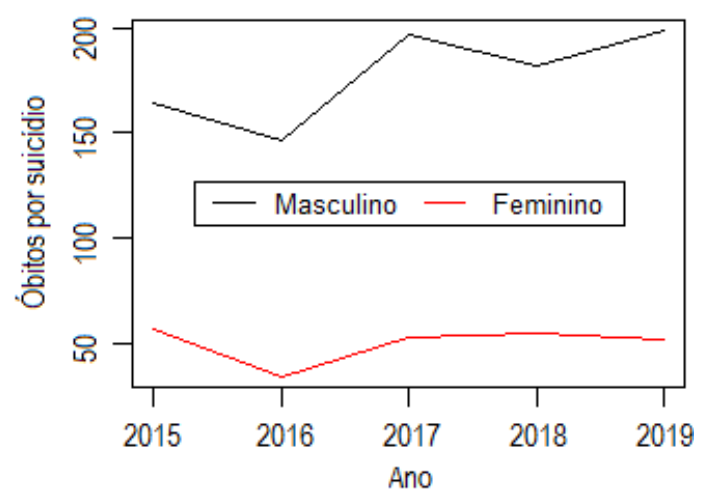

Fonte: Elaboração própria a partir de dados do SIM/DATASUS/MS.

No tocante ao número de óbitos por suicídio para o período de 2015 a 2019 na Figura 1, nota-se que no ano de 2016 houve uma redução em ambos gêneros e nos anos seguintes voltaram a crescer, principalmente entre o sexo masculino.

Na Figura 2 temos a distribuição da mortalidade por suicídio e gênero nas microrregiões de acordo com as mesorregiões.

Figura 2. Óbitos por suicídio e gênero nas microrregiões de acordo com as mesorregiões. Paraíba, Brasil, $2015-2019$.
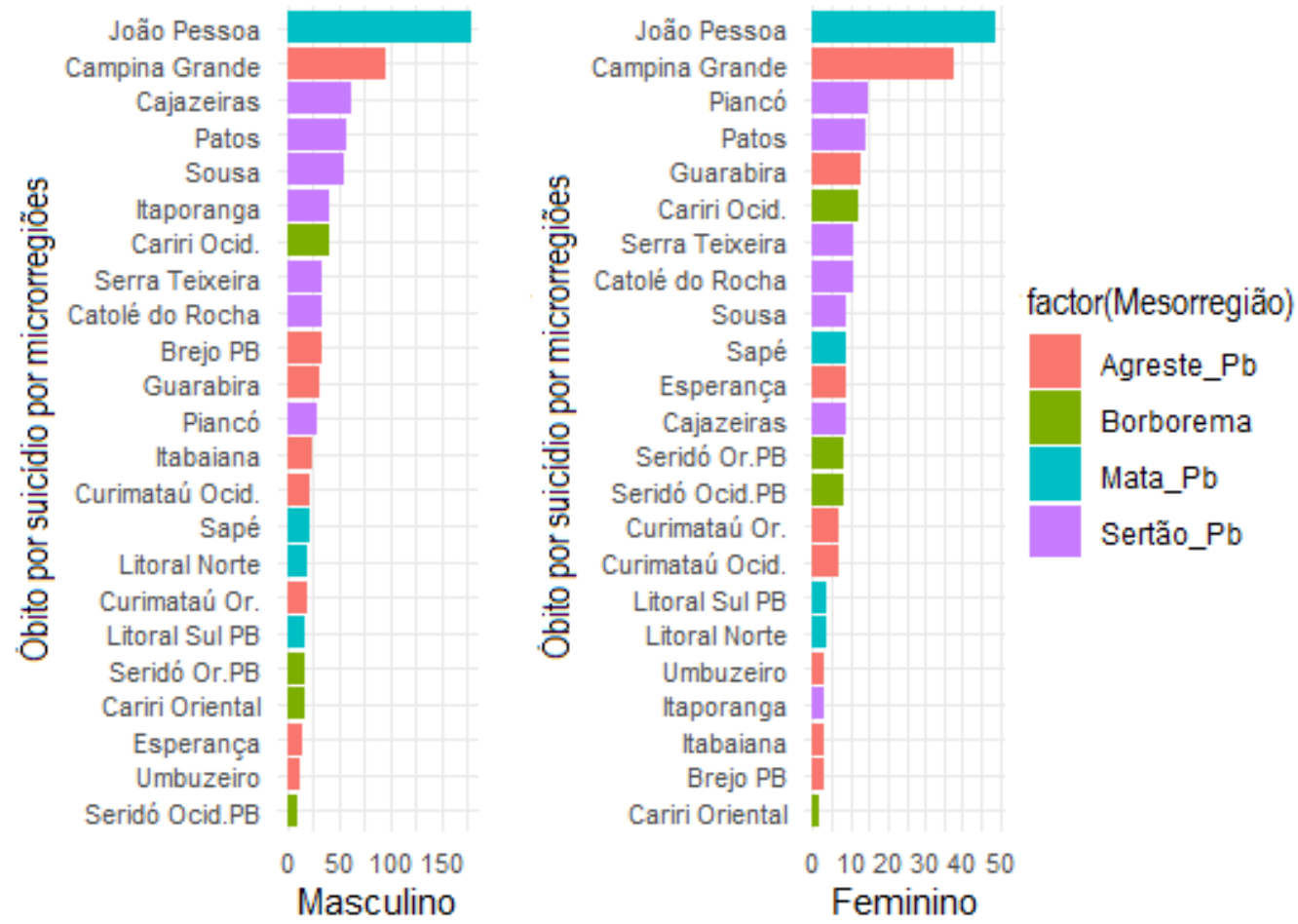

Fonte: Elaboração própria a partir de dados do SIM/DATASUS/MS. 
Observa-se na Figura 2 que as microrregiões de João Pessoa (20,0\%) e Campina Grande (11,8\%) lideram os casos de óbitos por suicídio no Estado da Paraíba, contrariamente, Umbuzeiro apresenta o menor número $(1,3 \%)$. Para os gêneros masculino e feminino, João Pessoa apresenta respectivamente 20,1\% e 19,5\%, enquanto Campina Grande 10,8\% e 15,1\%, respectivamente. Seridó Ocidental Paraibano apresenta o menor quantitativo masculino (1,0\%) de óbitos por suicídio e Cariri Oriental para o feminino com $0,8 \%$. Ao considerar as mesorregiões, Sertão Paraibano apresenta maior percentual de óbitos por suicídio com 33,6\%, Agreste Paraibano (29,8), Mata Paraibana $(26,6 \%)$ e por último a mesorregião da Borborema com 9,9\% dos casos.

A Figura 3 apresenta o percentual casos de óbitos por suicídio por raça, estado civil de acordo com o gênero.

Figura 2. Porcentagem de óbitos por suicídio, raça e estado civil de acordo com o gênero. Paraíba, Brasil, 2015 -2019.
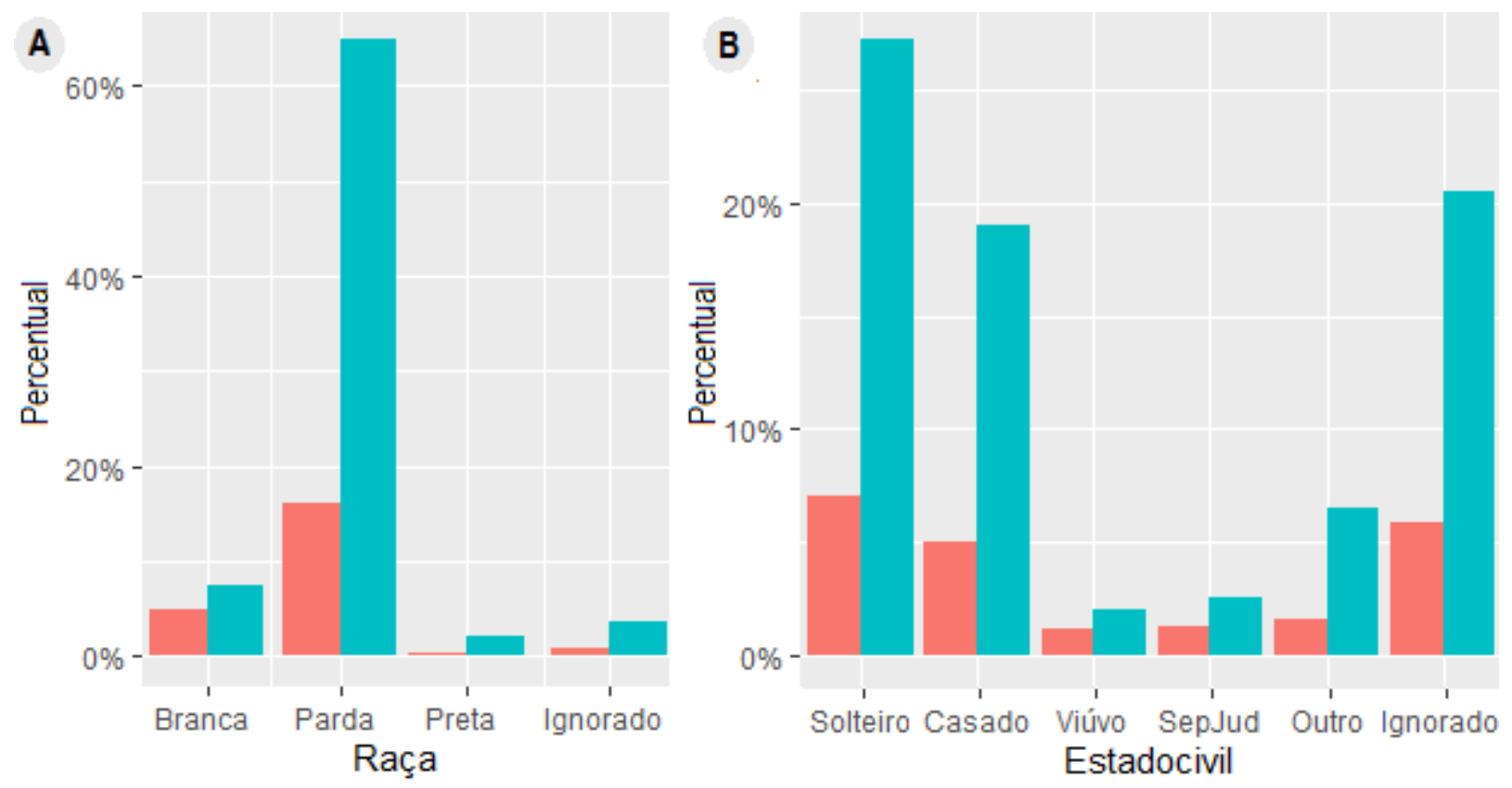

Gênero

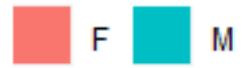

Fonte: Elaboração própria a partir de dados do SIM/DATASUS/MS.

Em conformidade com a Figura 2 (A) e Figura 2 (B), os óbitos por suicídio ocorreram predominantemente em homens pardos (+ de 60\%) e solteiros (+ de 25\%), também nota-se o mesmo perfil para as mulheres.

Estudos nacionais (Machado \& dos Santos, 2015; Franck et al., 2020; Silva et al., 2021) e estrangeiros (Fisher et al., 2015; Romero-Pimentel et al., 2018; Nunes, 2018; Kootbodien et al., 2020) também revelam maiores ocorrências de mortes por suicídio entre homens.

Um estudo no Ceará realizado por Lemos et al. (2021) analisou o perfil dos casos de suicídio no período de 2012 a 2019 e constatou que há uma prevalência no sexo masculino, em indivíduos pardos, solteiro, com idade compreendida entre 30 a 39 anos e escolaridade variando de 4 a 7 anos estudos. Em relação a faixa etária também corrobora com o achado neste estudo, conforme observa-se na Figura 3. 
Figura 3. Óbito por suicídio por gênero e ano de acordo com a faixa etária. Paraíba, Brasil, 2015-2019.
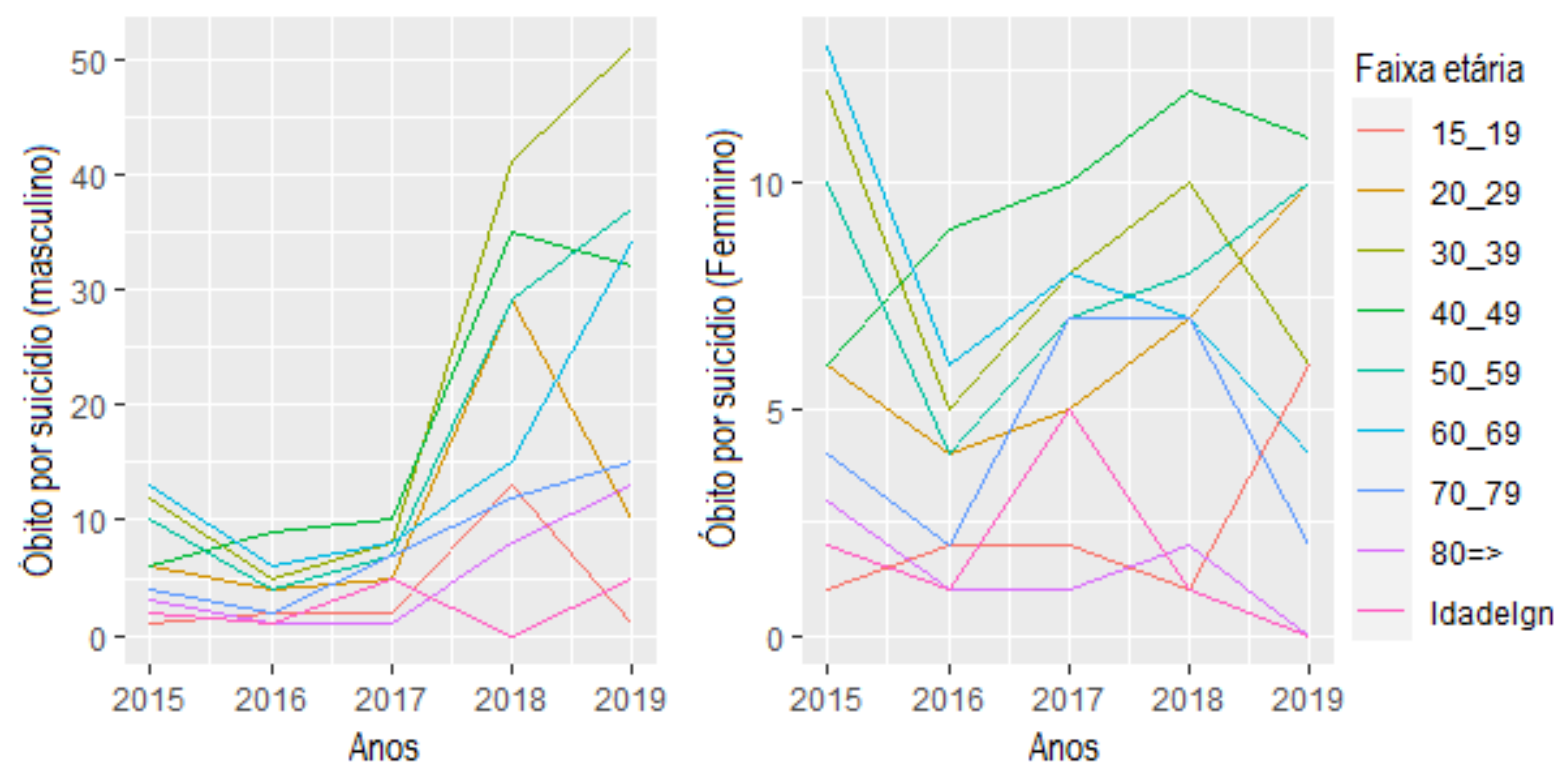

Fonte: Elaboração própria a partir de dados do SIM/DATASUS/MS.

Conforme observa-se na Figura 3, a mortalidade por suicídio ocorre principalmente em pessoas com idade ativa de trabalho de 20 a 59 anos $(73,4 \%)$. O gênero masculino com faixa etária de 30-39 anos (20,0\%) apresenta maior aumento contínuo de suicídio desde o ano de 2017, em seguida aparece os de 50-59 anos (17,0\%) e 60-69 (10,0\%) em menor número. Enquanto jovens de 20-29 anos (17,8\%) observou-se o maior declínio no número de morte autoprovocada seguido dos que tinham idade entre de 15-19 anos.

Em contrapartida, nota-se um aumento de suicídio entre o gênero feminino com faixa etária de 15 a 19 anos e na idade de 20 a 29 mais que dobrou entre os anos de 2016 a 2019; aumento significativo também entre mulheres de 50-59 anos. Em relação as mulheres de 40-49 anos são elas detentoras dos maiores números de suicídio desde o ano de 2016 no Estado, com uma leve diminuição em 2019 e mulheres de 70-79 anos tiveram a maior redução de mortalidade por suicídio de 2018 para 2019.

No que diz respeito aos anos de estudos, os que não tinham nenhuma instrução $(3,1 \%)$, de 1 a 3 anos de estudos $(4,2 \%)$, de 4 a 7 anos $(6,4 \%)$, de 8 a 11 anos (4,7\%), de 12 ou mais anos (4,2\%) e por fim, escolaridade ignorada apresentou elevadíssimo percentual de 77,4\%. Em homens, a mortalidade por suicídio ocorreu mais entre os que tinham de 4 a 7 anos de estudo $(6,3 \%)$ e para as mulheres predominou entre as que tinham 12 ou mais anos de escolaridade. Outros estudos também deparam-se com percentuais consideráveis de dados ignorados para o quesito escolaridade, tanto a nível nacional com $31 \%$ (Mata et al, 2020) como também para o Nordeste com o mesmo percentual de 31\% (Silva et al, 2021).

Na Figura 4, temos a distribuição percentual da mortalidade por suicídio nas microrregiões. 
Figura 4. Distribuição de óbitos por suicídio nas microrregiões. Paraíba, Brasil, 2015-2019.

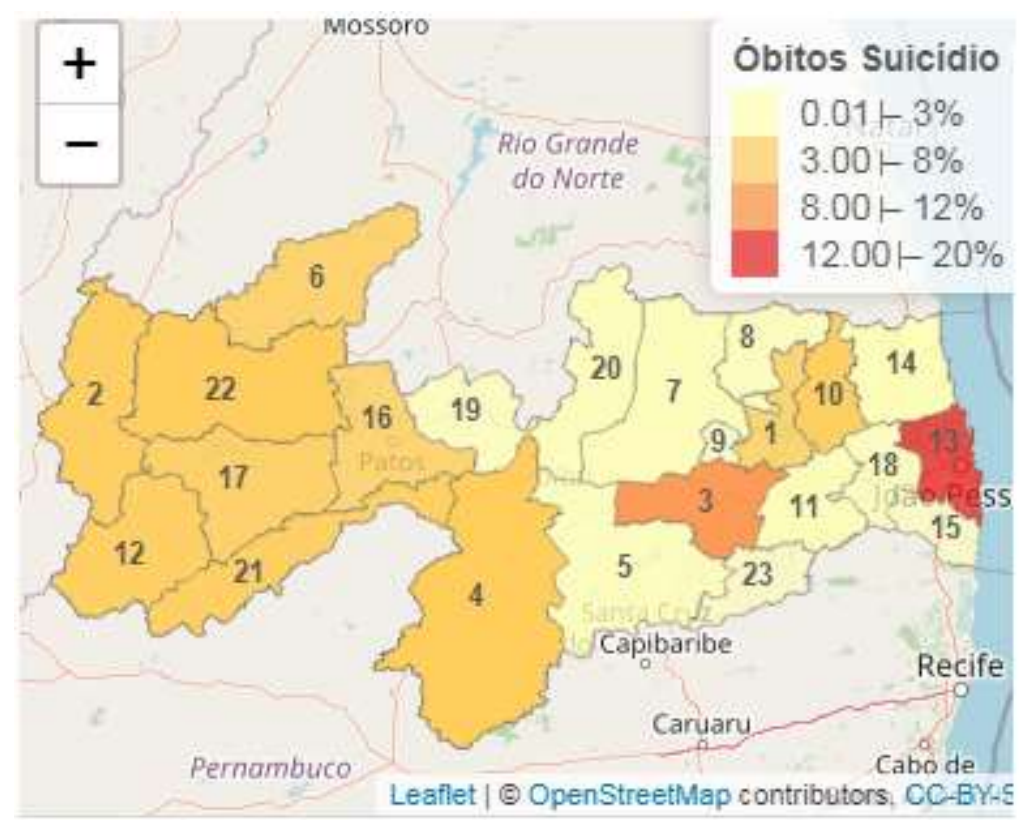

Obs.: Os números indicam as microrregiões:

$\begin{array}{llll}1=\text { Brejo Paraibano } & 7=\text { Curimataú Ocidental } & 13=\text { João Pessoa } & 19=\text { Seridó Ocidental } \\ 2=\text { Cajazeiras } & 8=\text { Curimataú Oriental } & 14=\text { Litoral Norte } & 20=\text { Seridó Oriental } \\ 3=\text { Campina Grande } & 9=\text { Esperança } & 15=\text { Litoral Sul } & 21=\text { Serra do Teixeira } \\ 4=\text { Cariri Ocidental } & 10=\text { Guarabira } & 16=\text { Patos } & 22=\text { Sousa } \\ 5=\text { Cariri Oriental } & 11=\text { Itabaiana } & 17=\text { Piancó } & 23=\text { Umbuzeiro } \\ 6=\text { Catolé do Rocha } & 12=\text { Itaporanga } & 18=\text { Sapé } & \end{array}$

Fonte: Elaboração própria a partir de dados do SIM/DATASUS/MS.

Observa-se na Figura 4 que os óbitos por suicídios no Estado da Paraíba estão mais concentrados nas microrregiões de João pessoa (12 a 20\%) e em seguida aparece Campina Grande (8 a 12\%). A cidade de João Pessoa, enquanto município mais populoso do Estado da Paraíba sozinha concentrou (14,1\%). Franck e colaboradores (2020) estudaram a mortalidade no Rio Grande do Sul no período de 2017 e 2018 e também identificou a capital Porto Alegre como sendo responsável pelo maior número de vítimas do Estado.

Também nota-se na Figura 4, que todas as microrregiões pertencentes a mesorregião do Sertão Paraibano $(2=$ Cajazeiras, 6 = Catolé do Rocha, 12 = Itaporanga, $16=$ Patos, $17=$ Piancó, Serra do Teixeira e $22=$ Sousa), a microrregião do Cariri Ocidental (4), Brejo Paraibano (1) e Guarabira (10) detém de 3 a $8 \%$ cada uma delas. E as demais microrregiões (5, 7, 8, $9,11,14,15,18,19,20$ e 23$)$ apresentam os menores percentuais $(0,01$ a $3 \%)$.

\section{Conclusão}

O suicídio é um problema de saúde pública tanto no Brasil como em todo o mundo e esta realidade tem contribuído para o aumento significativo das taxas de mortalidade nas unidades federativas.

Com este estudo, conclui-se que a mortalidade por suicídio no Estado da Paraíba apresenta tendência de crescimento e é marcada por cor e gênero. O perfil é majoritariamente definido por homens, pardos, solteiros, em idade ativa de trabalho com 20 a 59 anos, com ligeira predominância na faixa etária de 30 a 39 anos, 4 a 7 anos de estudos e residentes nas duas principais microrregiões de João Pessoa e Campina Grande, onde localiza-se as duas cidades mais populosas do Estado, João Pessoa e Campina Grande. 


\section{Referências}

Baére, F., \& Zanello, V. (2018, abr./jun). O gênero no comportamento suicida: Uma leitura epidemiológica dos dados do Distrito Federal. Estud. psicol. (Natal). vol.23 n $\mathrm{n}^{\circ} .2$. http://dx.doi.org/10.22491/1678-4669.20180017.

Bando, D., H., Brunoni, A., R., Fernandes, T. G., Benseñor, I. M., \& Lotufo, P. A. (2012). Suicide rates and trends in São Paulo, Brazil, according to gender, age and demographic aspects: a joinpoint regression analysis. Rev. Bras. Psiquiatr. 34(3). http://dx.doi.org/10.1016/j.rbp.2012.02.001.

Beringuel, B. M., Costa, H. V. V., Silva, A. P. S. C., \& Bonfim, C. V. (2020). Mortalidade por suicídio no Estado de Pernambuco, Brasil (1996-2015). Rev Bras Enferm. 73(Suppl 1):e20180270. http://dx.doi.org/10.1590/0034-7167-2018-0270.

Brasil. Ministério Público Federal. (2019). MPF e órgãos parceiros destacam importância

da prevenção ao suicídio na PB. Ministério Público Federal. [Internet]. Disponível em: http://www.mpf.mp.br/pb/sala-de-imprensa/noticias-pb/mpf-e-orgaosparceiros-destacam-importancia-da-prevencao-ao-suicidio-na-pb.

Brasil. Ministério da Saúde. (2017). Perfil epidiológico das tentativas e óbitos por suicídio no Brasil e a rede de atenção à saúde. Brasília.

Carmo, E. A., Ribeiro, B. S., Nery, A. A., \& Casotti, C. A. (2018). Tendência temporal da mortalidade por suicídio no Estado da Bahia. Cogitare Enferm. (23)2: e52516. http://dx.doi.org/10.5380/ce.v23i1.52516.

Cavalcante, F. G., Minayo, M. C. S., \& Mangas, R. M. N. (2013). Diferentes faces da depressão no suicídio em idosos. Ciênc. saúde coletiva 18 (10).

Cavanagh, J. T., Carson, A. J., Sharpe, M., \& Lawrie, S. M. (2003). Psychological autopsy studies of suicide: a systematic review. Psychological Medicine, 33(3):395-405.

Ceccon, R. F., Meneghel, S. N., Tavares, J. P., \& Lautert, L. (2014). Suicídio e trabalho metrópoles brasileiras: um estudo ecológico. Ciênc Saúde Coletiva. 19(7):2225-34. Doi: 10.1590/1413-81232014197.09722013

CEVS. Centro Estadual de Vigilância Saúde. (2018). Boletim de Vigilância Epidiológica de Suicídio e Tentativa de Suicídio. Rio Grande do Sul.

Chachamovich, E., Stefanello, S., Botega, N., \& Turecki, G. (2009). Which are the recent clinical findings regarding the association between depression and suicide?. Rev Bras Psiquiatr. 31(Suppl I):S18-25.

Cheng, J., Karambelkar, B., \& Xie, Y. (2021). leaflet: Create Interactive Web Maps with the JavaScript 'Leaflet' Library. R package version 2.0.4.1. https://CRAN.R-project.org/package=leaflet.

Fisher, L. B., Overholser, J. C., \& Dieter, L. (2015). Methods of committing suicide among 2,347 people in Ohio. Death Stud. 2015; 39(1):39-43. Doi: 10.1080/07481187.2013.851130.

Franck, M. C, Monteiro, M. G., \& Limberger, R. P. (2020). Suicide mortality in Rio Grande do Sul, Brazil: a crosssectional analysis of cases, $2017-2018$. Epidemiol Serv Saúde. 29(2):e2019512. https:// doi.org/10.5123/s1679-49742020000200014.

Kolves, K., \& De Leo, D. (2014). Regions with the Highest Suicide Rates for Children and Adolescents - Some Observations. J Child Adolesc Behav, 2(2):1000e104.

Kootbodien, T., Naicker, N., Wilson, K. S., Ramesar, R., \& London, L. (2020). Trends in suicide mortality in South Africa, 1997 to 2016. Int J Environ Res Public Health. 2020;17(6):1850. https://dx.doi. org/10.3390/ijerph17061850.

Lavor, M. L. S. S., Freitas, R. B., Souza, R. C., Silva, H. C. L., Azevedo-Neto, A. A., Nunes, M. C. S. L., \& Carvalho, A. L. B. (2020). Sazonalidade e tentativas de suicídio: comparativo entre a Paraíba, região nordeste e Brasil. Braz. J. Hea. Rev., Curitiba, v. 3, n. 3, p. 3960-3970. Doi:10.34119/bjhrv3n3-006.

Lemos, A. M., Jorge, M. S. B., \& Linard, C. F. B. M. (2021). Perfil do suicídio em um estado do nordeste brasileiro. Research, Society and Development, v. 10, n. 2, e45410212598. http://dx.doi.org/10.33448/rsd-v10i2.12598.

Macente, L. B., \& Zandonade, E. (2011). Estudo da série histórica de mortalidade por suicídio no Espírito Santo (de 1980 a 2006). J. Bras. Psiquiatr. 60(3). http://dx.doi.org/10.1590/S0047- 20852011000300001.

Machado, D. B., \& dos Santos, D. N. (2015). Suicídio no Brasil, de 2000 a 2012. J. Bras. Psiquiatr. 64(1). http://dx.doi.org/10.1590/0047-2085000000056.

Mata, K. C. R., Daltro, M. R., \& Ponde, M. P. (2020). Perfil epidemiológico de mortalidade por suicídio no Brasil entre 2006 e 2015. Revista Psicologia, Diversidade e Saúde, 9(1), 74-87. https://doi.org/10.17267/2317-3394rpds.v9i1.2842.

Nunes, A. M. (2018). Suicídio em Portugal: um retrato do país. J. bras. psiquiatr. 67 (1). https://doi.org/10.1590/0047-2085000000180.

OMS. Organización Mundial de Salud. (2018). Datos y crifras sobre el suicidio: infografía.

Ribeiro, J. M., \& Moreira, M. R. (2018). Uma abordagem sobre o suicídio de adolescentes e jovens no Brasil. Ciênc. saúde coletiva. v. 23, n. 9. Rio de Janeiro. Doi: 10.1590/1413-81232018239.17192018.

Romero-Pimentel, A. L., Mendoza-Morales, R. C., Fresan, A., Garcia-Dolores, F., Gonzalez-Saenz, E. E., Morales-Marin, M. E., Nicolini, H., \& Borges, G. (2018). Demographic and Clinical Characteristics of Completed Suicides in Mexico City 2014-2015. Frontiers in psychiatry, 9 , 402. https://doi.org/10.3389/fpsyt.2018.00402

Sehnem, S. B., \& Palosqui, V. (2014). Características epidemiológicas do suicídio no estado de Santa Catarina. Fractal, Rev. Psicol. 26(2). http://dx.doi.org/10.1590/1984-0292/624. 
Research, Society and Development, v. 10, n. 10, e196101018575, 2021

(CC BY 4.0) | ISSN 2525-3409 | DOI: http://dx.doi.org/10.33448/rsd-v10i10.18575

Silva, I. G., Maranhão, T. A., Silva, T. L., Sousa, G. J. B, Lira-Neto, J. C. G., \& Pereira, M. L. D. (2021). Diferenciais de gênero na mortalidade por suicídio. Rev Rene. 22:e61520. https://doi.org/10.15253/2175-6783.20212261520.

Teismann, T., Forkmann, T., Brailovskaia, J., Siegmann, P., Glaesmer, H., \& Margraf, J. (2018). Positive mental health moderates the association between depression and suicide ideation: A longitudinal study. International Journal of Clinical and Health Psychology. 18, 1-7.

https://doi.org/10.1016/j.ijchp.2017.08.001.

Wickham, H. (2016). ggplot2: Elegant Graphics for Data Analysis. Springer-Verlag New York.

WHO. World Health Organization. (2019). Suicide in the world - Global Health Estimates. World Health Organization: Geneva. 